\title{
Impact de la digitalisation sur la performance de financement des entreprises Marocaines
}

\author{
Mohamed HABACHI \\ Ph.D., Professeur, Laboratoire des études et recherches en sciences de gestion, \\ FSJES, Agdal, Université MED 5, Maroc
}

Abdelilah JEBBARI

Doctorant en sciences de gestion, Laboratoire des études et recherches en sciences de gestion, FSJES Agdal, Université MED 5, Maroc

\section{Salim EL HADDAD}

Doctorant en sciences de gestion, Laboratoire des études et recherches en sciences de gestion, FSJES Agdal, Université MED 5, Maroc

\begin{abstract}
Dans cet article détermine la relation positive entre la digitalisation et la performance du processus de financement des entreprises au Maroc. De ce fait, plusieurs variables ont été choisies pour expliquer la performance à savoir : les attitudes positives ou proactives par lesquelles la direction soutient le processus de financement (SD), la digitalisation, le comportement des entreprises à l'égard de la digitalisation du processus de demande de financement (CDC) et l'intensité concurrentielle (IC). Cette étude est basée sur des données collectées auprès d'un échantillon d'entreprises marocaines qui opèrent dans différents secteurs. Le traitement des données et l'analyse des relations de causalité entre les variables précédentes sont faits par les modèles d'équations structurelles estimés par l'approche PLS. L'étude a montré l'existence des relations positives entre les variables SD, digitalisation et performance du processus de financement alors que les variables CDC et IC n'ont pas d'effet sur la performance du processus de financement. Aussi, l'analyse des trois variables SD, digitalisation et performance du processus de financement, montre que la digitalisation modère positivement l'effet de la variable SD sur la performance du processus de financement.
\end{abstract}

Mots-clés : Digitalisation, crédit, modèles d'équations structurelles, PLS.

Digital Object Identifier (DOI): https://doi.org/10.52502/ijesm.v1i3.202 


\section{Introduction}

La crise sanitaire avait provoquée des mutations importantes des outils de travail et de gestion utilisés dans les différents domaines. En effet, les entreprises ont basculé de façon accélérée vers la digitalisation qui a permis de dépasser les contraintes de la crise covid-19 en garantissant la continuité d'activité en premier lieu dans les secteurs vitaux telles que le secteur bancaire, l'approvisionnement, l'enseignement, la santé, ....etc.

Pour les banques, le processus de digitalisation est un axe stratégique de développement depuis le début du siècle compte tenu des avantages procurés notamment dans la minimisation des coûts, la promotion des produits et l'agressivité commerciale. Cette orientation vise la mécanisation des taches et l'utilisation de la haute technologie pour la gestion de l'information notamment avec la généralisation de l'accès à l'internet. Plusieurs domaines ont été l'objet de ce changement de procédures comme le marketing, les ressources humaines, les crédits, la relation banque-client ...etc.

La dernière crise a accéléré la généralisation du processus de digitalisation dans les différents domaines notamment par la mise en place du travail à distance, de l'enseignement à distance et des services de santé à distance...etc. En effet, elle a permis de constater les avantages et les apports de la digitalisation. Toutefois, l'impact de ce processus sur la performance des activités doit faire l'objet d'une étude empirique confirmatoire. De ce fait, l'objectif de ce papier est d'étudier l'impact de la digitalisation sur la performance du processus de financement par des crédits bancaires au Maroc.

En se basant, sur une étude qualitative exploratoire, une enquête a été menée auprès d'un échantillon de clients et d'agences bancaires afin d'utiliser les modèles d'équations structurelles par la méthode PLS (partial last square) pour déterminer la relation entre la performance du processus de financement et la digitalisation.

Ce qui suit de ce papier est constitué de la revue de la littérature, la méthodologie ; ensuite, les résultats empiriques, la discussion et la conclusion..

\section{Revue de la littérature :}

La revue de la littérature montre que plusieurs recherches ont étudié l'impact de la technologie sur la création de la valeur et l'amélioration de la performance organisationnelle telles que Deltour et al. (2014), Chae et al. (2014) et Aral and Weill (2007), et que cette relation est influencée par d'autres facteurs tels que le niveau des ressources complémentaires et le support de la direction (Sheikh et al. , 2018).

Le marketing est l'un des domaines les plus concernés par l'impact de la digitalisation. En effet, la relation marketing-technologie a été étudiée par plusieurs chercheurs qui ont prouvé l'impact positif de la digitalisation et de l'internet tel que Kannan et Hongshuang, (2017). La relation digitalisationperformance en marketing est démontrée par de nombreux chercheurs tels que Brodie et al. (2007), Trainor et al. (2011), Bolos et al. (2016) et Olomu et al. (2016)

Pour la gestion des ressources humaines, diverses études ont traité l'apport de la nouvelle technologie, la digitalisation, l'internet et les réseaux sociaux sur l'attractivité des bons talents tel que Faliagka et al.(2012) qui ont montré que les demandeurs d'emplois utilisent de plus en plus l'internet et les sites de recherches d'emplois, Rafiq et al.(2013) qui ont constaté que suite à l'utilisation croissante de l'internet, les entreprises ont adopté l'environnement en ligne.

En ce qui concerne la relation entre la digitalisation et les activités bancaires, plusieurs recherches ont été menées telles que Shinta Winasis et al.(2020) qui ont étudié l'impact de la digitalisation sur l'engagement des employés dans le secteur bancaire indonésien, Ismail Musabegović et al.(2021) qui ont étudié l'interaction entre les technologies numériques et la performance des banques, Rila Anggraeni et al. (2021) qui ont examiné les facteurs influençant l'intention d'utiliser et l'utilisation des services bancaires numériques par les consommateurs, Meher et al.(2021) qui ont étudié l'impact de la banque 
numérique sur la croissance des micro-petites et moyennes entreprises en Inde, Kitsios et al.(2021) qui ont évalué le taux d'acceptation de l'e-service bancaire et Eyup Kahveci and Bert Wolfs (2018) qui ont étudié l'Impact de la banque numérique sur la performance des banques de dépôts.

Pour évaluer l'impact de la digitalisation sur la performance du processus de financement des entreprises Marocaines, cette étude utilise les modèles d'équations structurelles. En effet, la digitalisation sera considérée comme une variable médiatrice qui est le modèle le plus en plus utilisé par les chercheurs en gestion (Davcik ,2014). Les modèles d'équations structurelles ont été utilisé par divers chercheurs pour étudier la relation entre la digitalisation et la performance bancaire telles que Amin (2016), MBAMA (2018), Zuo et al.(2021) et Rila Anggraeni et al. (2021).

\section{Méthodologie}

Pour déterminer l'impact de la digitalisation sur la performance du processus de financement des entreprises, cette étude formule les hypothèses suivantes :

$\boldsymbol{H}_{\mathbf{1}}$ : Le support de la direction a une relation positive avec l'usage de l'internet dans le financement des entreprises.

$\boldsymbol{H}_{2}$ : Le support de la direction a une relation positive avec la performance du processus de financement des entreprises.

$\boldsymbol{H}_{3}$ : La digitalisation du processus de financement a une relation positive avec la performance du processus de financement.

$\boldsymbol{H}_{\mathbf{4}}$ : L'usage de la digitalisation par les demandeurs des crédits modère la relation entre le support de direction et la performance de financement.

$\boldsymbol{H}_{\mathbf{5}}$ : La pression de l'intensité concurrentielle modère la relation entre le support de direction et la performance de financement.

Le cadre de cette recherche est présenté par le schéma suivant :

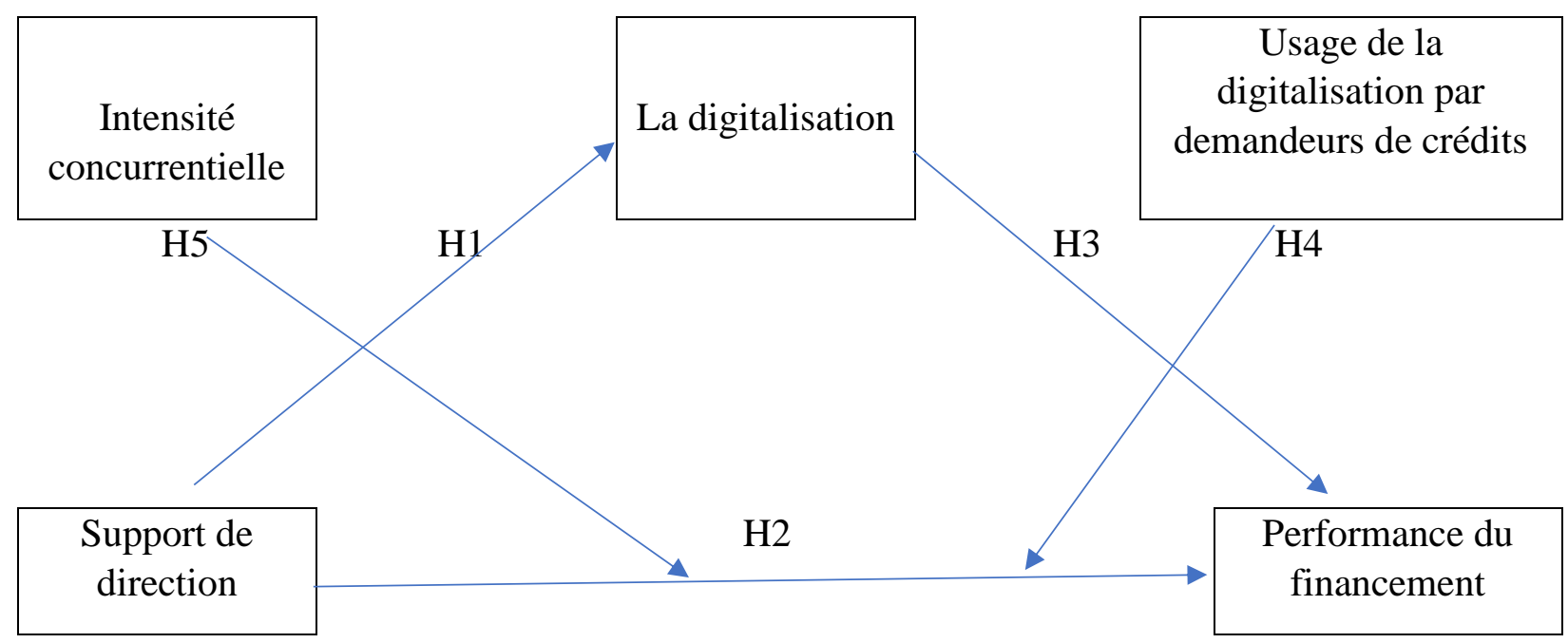

Figure 1 : Cadre de la recherche

\subsection{Choix de la méthode}

Pour vérifier la relation directe positive entre la digitalisation et la performance du financement, la digitalisation est considérée comme variable médiatrice et intermédiaire entre le support de direction et la performance de financement. Ensuite, l'éventuel rôle modérateur de l'usage de la digitalisation par les demandeurs de crédits et de l'intensité concurrentielle sur la performance est examiné

De ce fait, la modélisation est composée en quatre étapes à savoir :

- Etape 1 : Le développement du modèle conceptuel,

- Etape 2 : L'estimation des paramètres en fonction de l'algorithme choisi, 
- Etape 3 : L'évaluation du modèle,

- Etape 4 : Le réajustement du modèle en fonction des informations données par les indices d'évaluation afin d'obtenir le meilleur modèle possible.

L'estimation des paramètres du modèle se fera, en général, soit par la méthode Lisrel (Linear Structural Relationships) qui repose sur l'analyse de la structure de covariance et utilise l'approche du maximum de vraisemblance ; elle requiert la multi-normalité des données, soit, par la méthode PLS (Partial Least Square) qui repose sur l'analyse de la variance et utilise l'approche des moindre carrés partiels. Cette dernière qui sera utilisée dans ce papier a été développée par Wold (1973, 1980a, 1980b).

Les modèles d'équations structurelles selon Roussel et al. (2005), permettent :

- L'analyse simultanée des effets linéaires qui sont supposés relier plusieurs variables latentes indépendantes et dépendantes ;

- L'analyse d'effets conjoints sur plusieurs variables dépendantes ;

- Les tests de validité des construits, de fiabilité et de validité interne d'instruments tels que les échelles d'attitudes ...etc.

Le choix de la méthode PLS est dicté par les contraintes de déroulement de l'enquête. En effet, si la taille de l'échantillon est faible, l'approche PLS est recommandée pour estimer les modèles d'équations structurelles (Chin et al.(1999)).

\subsection{Présentation des modèles d'équations structurelles.}

Les modèles d'équations structurelles utilisent deux types de variables :

- La variable manifeste : est une variable pour laquelle une mesure peut être directement recueillie (observée, mesurée, etc.).

- La variable latente : correspond à une caractéristique qui n'est pas directement observable et qui ne peut pas être mesurée directement.

Un modèle d'équations structurelles à variables latentes consiste en un système d'équations dites structurelles pouvant être représentées par un graphe orienté. Chaque variable manifeste est associée à une seule variable latente et il existe des relations entre les variables latentes. Dans le cadre de l'utilisation de variables latentes, on sépare généralement le modèle en deux sous-modèles :

- Le modèle de mesure ou modèle externe : est une sous-partie du modèle complet incluant les relations entre les variables manifestes et latentes.

- Le modèle structurel ou modèle interne : est une sous-partie du modèle complet incluant les relations entre les variables latentes.

\subsection{Les modèles externes.}

Les modèles externes peuvent être des modèles formatifs ou réflectifs. La distinction entre les deux dépend du schéma liant la variable latente et la variable manifeste :

Pour le modèle formatif utilisé dans cette étude, on suppose que chaque variable latente est une combinaison linéaire de ses variables manifestes correspondantes. En effet, chaque variable latente $A_{k}$ est en relation avec l'ensemble des variables manifestes $X_{k i}, i=1, \ldots, p$ qui lui sont associées :

Avec

$$
A_{k}=\sum_{i=1}^{p} b_{k i} X_{k i}+\delta_{k}
$$

$b_{k i}$ est un poids et $\delta_{k}$ est un vecteur d'erreur qui est supposé de moyenne nulle et non corrélé aux $X_{k i}$.

\subsection{Les modèles internes.}

Il est défini par des équations linéaires reliant les variables latentes. Pour toute $A_{k}$ endogène, 
on a :

$$
A_{k}=\sum_{i=1}^{q} \beta_{k i} A_{i}+\tau_{k}
$$

Avec

$$
\begin{array}{ll}
\checkmark & i=1, . ., \mathrm{q} \text { nombre de } A_{i} \text { exogène ayant relation avec } A_{k} \\
\checkmark & \operatorname{Cov}\left(\tau_{k}, A_{i}\right)=0, \forall i \neq k \\
\checkmark & \operatorname{Cov}\left(\tau_{k}, \varepsilon_{k j}\right)=0 \forall k=1,0, \cdots K \text { et } \forall i=1,2, \cdots q
\end{array}
$$

où $\beta_{k i}$ représente le coefficient structurel associé à la relation entre les variables $A_{k}$ et $A_{i}$, et $\tau_{k}$ est un terme d'erreur associé à la variable latente endogène $A_{k}$

\subsection{Estimation interne et externe des variables latentes.}

L'estimation externe notée $\boldsymbol{A}_{\boldsymbol{k}}^{*}$ des variables latentes standardisées sont égale à la combinaison linéaire de leurs variables manifestes centrées :

$$
\boldsymbol{A}_{\boldsymbol{k}}^{*} \propto \mp X_{k} w_{k}=\sum_{i=1}^{p} X_{k i} w_{k i}
$$

où le symbole $\propto$ signifie que le terme de gauche est égal au terme de droite standardisé et \pm montre l'ambiguïté du signe. On choisit le signe de façon à ce que $\boldsymbol{A}_{\boldsymbol{k}}^{*}$ soit positivement corrélée avec le plus grand nombre possible de colonnes de $X_{k}$. Les éléments $w_{k}$ sont appelés poids externes.

L'estimation interne de $\boldsymbol{A}_{\boldsymbol{k}}^{* *}$ est définie par :

$$
\boldsymbol{A}_{\boldsymbol{k}}^{* *} \propto \sum_{i=1}^{q} h_{k i} l_{k i} \boldsymbol{A}_{\boldsymbol{i}}^{*}
$$

où $H=\left(h_{k i}\right), i=1, \ldots, q$ est une matrice de 0 et de 1 telle que $h_{k i}=1$ si $A_{i} \rightarrow A_{k}$ ou $A_{k} \rightarrow$ $A_{i}$ et $L=\left(l_{k i}\right)$ est la matrice des poids internes définie par trois méthode à savoir la méthode Centroïde, la méthode Factorielle et la méthode Structurelle.

A partir de cette étape, il existe deux façons de mettre à jour les poids externes $w_{k i}$ à savoir la méthode A et la méthode B. Pour le choix de la méthode, Wold (1982) a associé au mode $\mathrm{A}$, le modèle réflectif et au mode $\mathrm{B}$, le modèle formatif. Cette formalisation est la plus répandue dans la pratique mais n'a de fondement mathématique robuste. En pratique, les modes sont associés à l'objet de l'étude. Ainsi, le choix du mode pourra être motivé par différents facteurs:

- si l'étude veut donner un poids plus important au modèle externe, il faut utiliser le mode A, - si l'étude veut donner un poids plus important au modèle interne, il faut utiliser le mode B, - si il y a beaucoup de variables manifestes par bloc, il faut favoriser le mode A.

\subsection{La validité des modèles de mesure}

La variance extraite moyenne (AVE) peut être utilisée comme un test de validité convergente et divergente. L'AVE reflète la communalité moyenne de chaque facteur latent dans un modèle de réflexion.

Dans un modèle adéquat, l'AVE doit être supérieure à 0.5 (Chin, 1998 ; Höck and Ringle, 2006) et supérieure aux charges croisées, ce qui signifie que les facteurs doivent expliquer au moins la moitié de la variance de leurs indicateurs respectifs. Un AVE inférieure à 0,50 signifie que la variance des erreurs est supérieure à la variance expliquée (Fornell and Larcker, 1981).

La validité du modèle de mesure est faite par deux étapes :

- La première étape concerne la validité discriminante est mesurée grâce au critère de Fornell et Larcker (1981). Celui-ci indique que le construit doit partager plus de 
variance avec ses indicateurs qu'avec tout autre construit (Hair, Sarstedt, Hopkins, and Kuppelwieser, 2014).

- La deuxième étape de la validité renvoie au degré de différenciation d'un construit à un autre, en examinant leur potentiel de chevauchement ou de croisement (cross loading).

\subsection{La validité du modèle structurel.}

Pour évaluer les modèles structurels, il faut évaluer les relations hypothétiques dans le modèle structurel. De ce fait, il faut déterminer :

(1) L'effet direct via les critères de Hair et al. (2014),

(2) L'effet indirect, incluant la médiation (technique de ré-échantillonnage bootstrapping).

(3) La modération de variable modératrice.

Les critères d'évaluation utilisés dans cette étude sont ceux définis par Hair et al. (2014) à savoir:

\section{i- Le coefficients de trajectoire :}

Ces coefficients représentent les relations hypothétiques entre les constructions. La signification de cette relation dépend de la p-value qui doit être inférieur à 5\% selon Henseler, Ringle et Sarstedt (2014), pour juger l'importance de la relation on utilise la valeur statistique $T$ qui doit être, selon les recommandation de Hair et al.(2014), supérieure à 1,64 pour une relation importante.

\section{ii- $\mathbf{R}^{2}$ multiples :}

Il mesure le comportement prédictif du modèle. En effet, Si R ${ }^{2}$ est supérieur à $10 \%$ alors le modèle est significatif. Si $\mathrm{R}^{2} \in[5 \%, 10 \%]$, alors le modèle est tangent et si $\mathrm{R}^{2}$ est inférieur à 0,05 , alors le modèle n'est pas significatif.

\section{iii- Taille de l'effet $\left(F_{k}^{2}\right)$ :}

La qualité du modèle structurel (modèle interne) est évaluée par la statistique $F_{k}^{2}$ appelée la redondance (redundancy) qui évalue la qualité du modèle structurel pour chaque bloc endogène $\mathrm{k}$ en prenant en compte le modèle de mesure. Elle est définie par

$$
F_{k}^{2}=H^{2} \times R^{2}\left(A_{k}^{*},\left[\text { les } A_{i}^{*} \text { qui expliquent } A_{k}^{*}\right]\right)
$$

Elle part de la même idée que la communauté mais la variable latente est remplacée par son estimation à partir des variables latentes voisines.

La taille de l'effet de la construction omise pour une construction endogène particulière peut être déterminée telle que $0,02,0,15$ et 0,35 représentent respectivement des effets faibles, moyens et importants (significatifs).

iv- Le coefficient $\mathbf{Q}^{2}$ de Stone-Geisser (indice de redondance en validation croisée):

L'objectif du coefficient de Stone-Geisser $\left(\mathrm{Q}^{2}\right)$ est le test de la validation croisée entre les variables manifestes d'une variable latente endogène et l'ensemble des variables manifestes des 
variables latentes expliquant ladite variable latente endogène en utilisant le modèle structurel estimé (Bennaceur and Chafik, 2019).

Pour une variable latente $A_{k}$, le coefficient $Q^{2}$ de Stone-Geisser est calculé par :

$$
Q_{k}^{2}=1-\frac{\sum_{j=1}^{k p} \sum_{i=1}^{N}\left(x_{k j i}-\overline{x_{k J}}-m_{k j}^{*} \operatorname{pred}\left(\boldsymbol{A}_{\boldsymbol{k}}^{*}\right)\right)^{2}}{\sum_{j=1}^{k p} \sum_{i=1}^{N}\left(x_{k j i}-\overline{x_{k J}}\right)^{2}}
$$

où $x_{k j i}$ est la i-éme e observation de la j-ème variable du bloc $k, m_{k j}^{*}$ est l'estimateur de $m_{k j}$ et $\operatorname{pred}\left(\boldsymbol{A}_{\boldsymbol{k}}^{*}\right)=\sum_{\boldsymbol{i}, A_{i} \rightarrow A_{k}} \boldsymbol{\beta}_{\boldsymbol{i}}^{\prime} \boldsymbol{A}_{\boldsymbol{i}}^{*}$, la prédiction de la variable latente $A_{k}$ obtenue à partir des variables latentes qui l'expliquent dans le modèle structurel.

Si $Q_{k}^{2}>0$ on dit que le modèle présente une validité prédictive (Fernandes, 2012).

La qualité du modèle de mesure pour chaque bloc est mesurée par la communauté. La qualité de chaque équation structurelle est calculée par l'indice $Q^{2}$ de Stone-Geisser. Les niveaux de significativité peuvent être calculés en le test t de Student ou en utilisant des méthodes comme le bootstrap ou le jackknife .

\section{v-La statistique $\boldsymbol{H}^{\mathbf{2}}$}

La qualité du modèle de mesure (modèle externe) est évaluée par la statistique $H^{2}$ appelée la communauté (communality) définie par :

$$
H^{2}=\frac{\sum_{i=1}^{k p} \operatorname{cor}^{2}\left(X_{k i}, \boldsymbol{A}_{\boldsymbol{k}}^{*}\right)}{k p}
$$

Elle représente la proportion de la variance des variables manifestes expliquée par leur variable latente associée.

vi- L'Indice d'ajustement GoF (Goodness-of-fit) :

C'est un indice de validation globale. Il st égal à la moyenne géométrique à la fois de l'AVE et la moyenne de $\mathrm{R}^{2}$ des variables endogènes (Jörg and Marko, 2013) .

$$
G o F=\sqrt{\overline{H^{2}} \times \overline{R^{2}}}
$$

Le GOF doit être supérieure à 0,25 pour être considéré comme moyen, et supérieur à 0,36 pour être considéré comme très large selon Wetzels et al.(2009)

\subsection{Définition des construits.}

La définition des construits est déterminée à partir de la revue de littérature comme suit :

- Digitalisation (E-financement) : Un nouveau processus en matière de financement qui consiste à utiliser l'internet pour initier et constituer le dossier de crédit.

- Performance financement : Trois dimensions : efficience, efficacité et adaptabilité. L'objectif est de garantir l'attractivité de la banque et la fidélité des clients.

- Support de la direction : Les attitudes positives ou proactives auquel la direction soutient le processus d'octroi de crédit par l'allocation des ressources et des moyens nécessaires.

- Le comportement des demandeurs des crédits à l'égard de la digitalisation : L'impact de l'environnement numérique sur l'adoption et l'usage de la digitalisation par les demandeurs de financement.

- L'intensité concurrentielle : l'agressivité commerciale des concurrents dans un marché de crédits qui peut être amortis par l'adoption de la digitalisation du processus de crédits. 


\subsection{Définition des items de mesure :}

Les items de mesures sont définis dans le tableau 1 suivant; ils sont empruntés de la revue de littérature mentionnée dans le tableau 1 et opérationnalisés par le biais d'échelles de likert en sept points :

Tableau 1 : définition des ithems

\begin{tabular}{|c|c|}
\hline Construit & Items \\
\hline $\begin{array}{l}\text { Digitalisation }(\boldsymbol{E F}) \\
\text { items utilisés en marketing adaptés aux } \\
\text { processus de crédit bancaire. }\end{array}$ & $\begin{array}{l}\text { EF1 : Votre banque utilise les ressources internet (site, } \\
\text { web, e-mail, réseaux sociaux,...) pour octroyer des crédits. } \\
\text { EF2 : Votre banque utilise les ressources de digitalisation } \\
\text { comme support de communication pour promouvoir les } \\
\text { activités de crédits (grille de taux, simulation, condition de } \\
\text { paiement, garanties) } \\
\text { EF3 : Votre banque utilise les ressources internet (réseaux } \\
\text { sociaux, job board, site de l'entreprise) pour recevoir des } \\
\text { feeds back des clients de crédits. } \\
\text { EF4 : Votre banque utilise les ressources internet pour } \\
\text { faciliter l'octroi de crédits } \\
\text { EF5 : Votre banque possède une infrastructure et des } \\
\text { compétences pour implémenter la digitalisation (serveur } \\
\text { pour stockage de données, une équipe informatique, sous } \\
\text { traitance) } \\
\text { EF6 : Votre banque affecte un budget pour le } \\
\text { développement de la digitalisation }\end{array}$ \\
\hline $\begin{array}{l}\text { Performance financement ( } \boldsymbol{P F} \text { ) } \\
\text { items utilisés en marketing adaptés au } \\
\text { financement bancaire. } \\
\text { Brodie et al., (2007), Chavey (2010), Morgan } \\
\text { (2012) et Trainor et al. (2011) }\end{array}$ & $\begin{array}{l}\text { Pf1 : Internet à un effet important sur l'efficacité du } \\
\text { processus d'octroi de crédit. } \\
\text { Pf2 : Internet permet d'attirer et de sélectionner les clients } \\
\text { solvables et de réussir l'action de de financement } \\
\text { Pf3 : Internet a un effet positif sur la fidélisation des } \\
\text { clients de crédits }\end{array}$ \\
\hline $\begin{array}{l}\text { Support de la direction }(\boldsymbol{S D}) \\
\text { items utilisés en marketing adaptés aux } \\
\text { processus de financement bancaire. } \\
\text { Germann et al. (2013) et Sheikh et al. (2018) }\end{array}$ & $\begin{array}{l}\text { S1 : Votre top management a une attitude favorable pour } \\
\text { l'utilisation d'internet dans le processus d'octroi de crédits } \\
\text { S2 : Votre top management a conscience des bénéfices à } \\
\text { utiliser les TIC au financement. } \\
\text { S3 : Votre top management considère les canaux d'internet } \\
\text { comme leviers pour l'attraction des clients solvables } \\
\text { S4 : Votre top management encourage l'utilisation de } \\
\text { l'internet dans le processus d'octroi de crédits. }\end{array}$ \\
\hline $\begin{array}{l}\text { Le comportement des demandeurs de crédits à } \\
\text { l'égard de la digitalisation ( } \boldsymbol{C} \boldsymbol{D} \boldsymbol{C}) \\
\text { items utilisés en marketing adaptés au } \\
\text { financement bancaire. } \\
\text { Jaworski and Kohli, (1993) et Kannan and } \\
\text { Hongshuang( 2017) }\end{array}$ & $\begin{array}{l}\text { CD1 : Les demandeurs de crédit utilisent internet d'une } \\
\text { manière intense } \\
\text { CD2 : Les demandeurs de crédits préfèrent les } \\
\text { consultations via internet } \\
\text { CD3 : Les demandeurs de crédits s'informent et change } \\
\text { avec la banque via les canaux internet }\end{array}$ \\
\hline $\begin{array}{l}\text { L'intensité concurrentielle (IC) } \\
\text { items utilisés en marketing adaptés au } \\
\text { financement bancaire. } \\
\text { Porter and Millar, (1985) et (Zhu et al.(2006) }\end{array}$ & $\begin{array}{l}\text { IC1 : L'usage d'internet dans le financement est très } \\
\text { intense dans le secteur bancaire. } \\
\text { IC2 : Internet est utilisé intensément dans le financement } \\
\text { et par le secteur bancaire } \\
\text { IC } 3 \text { : L'utilisation d'internet est un avantage compétitif } \\
\text { pour le financement des bons clients }\end{array}$ \\
\hline
\end{tabular}

\section{Etude empirique.}

\subsection{Présentation de l'échantillon.}

L'étude empirique est faite sur un échantillon de 74 entreprises répartie dans plusieurs secteurs. En effet, 2,5\% sont des sociétés de télécommunication, 7,5\% sont des entreprises de 
BTP, $14 \%$ sont des établissements de formation, $6 \%$ opèrent dans le secteur de négoce, $13 \%$ dans l'industrie, 29,6\% dans les services, 17,1\% dans l'agroalimentaire et $16 \%$ dans différents secteurs.

\subsection{Evaluation des modèles de mesures}

L'analyse des données par le logiciel smartPLS a permis de déterminer les modèles de mesure. De ce fait, Les équations des modèles de mesure pour chaque variable latente se présentent comme suit :

- Digitalisation (EF)

$$
\left\{\begin{array}{l}
\mathbf{E F 1}=0,901 * \mathbf{E F}+\varepsilon_{11} \\
\mathbf{E F 2}=0,825 * \mathbf{E F}+\varepsilon_{12} \\
\mathbf{E F 3}=0,799 * \mathbf{E F}+\varepsilon_{13} \\
\mathbf{E F 4}=0,830 * \mathbf{E F}+\varepsilon_{14} \\
\mathbf{E F 5}=0,963 * \mathbf{E F}+\varepsilon_{15} \\
\mathbf{E F 6}=0,840 * \mathbf{E F}+\varepsilon_{16}
\end{array}\right.
$$

- Performance de financement $(P F)$

$$
\left\{\begin{array}{l}
\mathbf{P f 1}=0,901 * \mathbf{P F}+\varepsilon_{21} \\
\mathbf{P f} 2=0,932 * \mathbf{P F}+\varepsilon_{22} \\
\mathbf{P f} 2=0,917 * \mathbf{P F}+\varepsilon_{23}
\end{array}\right.
$$

\section{- Support de la direction $(S D)$}

$$
\left\{\begin{array}{l}
\mathbf{S} 1=0,918 * \mathbf{S D}+\varepsilon_{31} \\
\mathbf{S} 2=0,903 * \mathbf{S D}+\varepsilon_{32} \\
\mathbf{S 3}=0,926 * \mathbf{S D}+\varepsilon_{33} \\
\mathbf{S} 4=0,861 * \mathbf{S D}+\varepsilon_{34}
\end{array}\right.
$$

- Le comportement des demandeurs de crédit à l'égard de la digitalisation $(C D C)$ :

$$
\left\{\begin{array}{l}
C D 1=0,902 * C D C+\varepsilon_{41} \\
C D 2=0,915 * C D C+\varepsilon_{42} \\
C D 3=0,850 * C D C+\varepsilon_{43}
\end{array}\right.
$$

\section{- L'intensité concurrentielle (IC):}

$$
\left\{\begin{array}{l}
\text { IC } \mathbf{1}=0,918 * \boldsymbol{I C}+\varepsilon_{51} \\
\boldsymbol{I C} \mathbf{2}=0,888 * \boldsymbol{I C}+\varepsilon_{52} \\
\boldsymbol{I C} \mathbf{3}=0,916 * \boldsymbol{I C}+\varepsilon_{53}
\end{array}\right.
$$

La validité des modèles de mesures est faite par trois élément que sont Cronbach Alpha, Fiabilité du composite et Variance moyenne extraite. En effet, pour la digitalisation le Cronbach Alpha est égale à 0,940 , la Fiabilité du composite (CR) est égale à 0,960 et la variance moyenne extraite (AVE) est égale à 0,812 ). Pour la performance Du financement bancaire, le Cronbach Alpha est égale à 0,908 , la Fiabilité du composite $(\mathrm{CR})$ est égale à 0,938 et la variance moyenne extraite (AVE) est égale à 0,949). Pour le Support de la direction, le Cronbach Alpha est égale à 0,943 , la Fiabilité du composite (CR) est égale à 0,956 et la variance moyenne extraite (AVE) est égale à 0,868). Pour Le comportement des demandeurs de crédits à l'égard de la digitalisation, le Cronbach Alpha est égale à 0,930 , la Fiabilité du composite (CR) est égale à 0,901 et la variance moyenne extraite (AVE) est égale à 0,865)et pour l'intensité concurrentielle, le Cronbach Alpha est égale à 0,880, la Fiabilité du composite (CR) est égale à 0,935 et la variance moyenne extraite (AVE) est égale à 0,828 ). 
La validité convergente (fiabilité du composite) sont supérieures à la valeur seuil de 0.7 ce qui signifié la fiabilité des items. La validé discriminante (AVE) est entre 0,812 et 0,949 pour tous les construis ce qui signifie qu'ils remplissent la condition statistique, qui est d'être supérieure à 0,5 . De ce fait, les échelles de mesure sont valides. Le calcul de $\mathrm{R}^{2}$ des variables latentes endogènes présenté dans la suite montre que la variable digitalisation est expliquée par $87,6 \%$ de la variable support de direction et la variable performance de financement est expliquée par $95,6 \%$ de la variable digitalisation.

La validité discriminante de l'ensemble des construits est vérifié par le critère de Fornell et Larcker. Les résultats sont présentés dans le tableau 2 suivant :

Tableau 2 : Critère de Fornell et Larcker

\begin{tabular}{|c|c|c|c|c|c|}
\hline & $C D C$ & $E F$ & $I C$ & $P F$ & $S D$ \\
\hline$C D C$ & 0,901 & & & & \\
\hline$E F$ & 0,697 & 0,896 & & & \\
\hline$I C$ & 0,889 & 0,809 & 0,903 & & \\
\hline$P F$ & 0,635 & 0,805 & 0,776 & 0,925 & \\
\hline$S D$ & 0,669 & 0,804 & 0,807 & 0,905 & 0,936 \\
\hline
\end{tabular}

Le critère de Fornell et Larcker (1981) confirme la validité discriminante de l'ensemble des construits composant le modèle du fait que la variance extraite moyenne (AVE) des trois construits est supérieure au carré des corrélations de ces construits avec les autres variables latentes du modèle.

Le tableau 1 de l'annexe 1, confirme la validité discriminante des variables latentes, car celles-ci ne se chevauchent pas et partagent plus de variance avec leurs propres indicateurs qu'avec d'autres variables latentes.

\section{3. Évaluation du modèle structurel}

Les équations structurelles entre les variables latentes sont définies comme suit :

$$
\left\{\begin{array}{l}
\mathrm{EF}=0,924 * \mathrm{SD}+\tau_{\mathrm{k}} \\
\mathrm{PF}=0,557 * \mathrm{SD}+0,504 * \mathrm{EF}-0,068 * \mathrm{CDC}-0,032 * \mathrm{IC}+\tau_{2}
\end{array}\right.
$$

Pour évaluer les coefficients de trajectoire $\left(\beta_{i}\right)$, les valeurs de la statistique $T$ et le coefficient des régressions des 74 réponses, il faut effectuer 5000 des itérations (Hair et al., 2014). Cette étude contient trois hypothèses basées sur le lien direct des relations.

L'analyse des tests basés sur la statistique $T$ montre que les relations définies par les hypothèses $\mathrm{H}_{1} \mathrm{H}_{2} \mathrm{H}_{3}$ sont significatives et les hypothèses sont confirmées, du fait que la statistique $T$ qui est égal respectivement à 67,$93 ; 6,87$ et 5,99 sont supérieurs à 1,64 avec une forte significativité dans la relation entre le support de la direction et la digitalisation. En conséquence, le support de direction a un impact positif sur la digitalisation et la performance de financement, et que la digitalisation a un impact positif sur la performance de financement.

En revanche, les hypothèses $H_{4}, H_{5}$ ne sont pas confirmées du fait que la statistique test $T$ est inférieur à 1,64 . 
Les résultats des tests statistiques basés sur la statistique $T$ sont représentés dans le tableau 3 suivant :

Tableau 3 : Détermination des BETA et des résultats des tests.

\begin{tabular}{|l|c|c|c|c|}
\hline \multicolumn{1}{|c|}{ Relation } & BETA & Écart-type & Valeur T & valeurs-p \\
\hline$H_{1}: S D->E F$ & 0,924 & 0,014 & 67,93 & 0,000 \\
\hline$H_{2}: S D->P F$ & 0,547 & 0,081 & 6,87 & 0,000 \\
\hline$H_{3}: E F->P F$ & 0,504 & 0,086 & 5,99 & 0,000 \\
\hline$H_{4}: C D C->P F$ & $-0,066$ & 0,049 & 1,37 & 0,166 \\
\hline$H_{5}: I C->P F$ & $-0,033$ & 0,064 & 0,49 & 0,622 \\
\hline
\end{tabular}

Pour le coefficient $\mathrm{R}^{2}$ des variables aléatoires endogènes, les valeurs du $R^{2}$ des deux construits dépendants, présentées dans le tableau 4 suivant, sont supérieur à 0,67 , qui selon Chin (1998) est un résultat significatif

Tableau 4 : les valeurs de $R^{2}$

\begin{tabular}{|c|c|c|}
\hline & $\mathrm{R}^{2}$ & Résultat \\
\hline$E F$ & 0,877 & Le modèle est significatif \\
\hline$P F$ & 0,988 & Le modèle est significatif \\
\hline
\end{tabular}

Les valeurs de $F_{k}^{2}$ présentées dans le tableau 2 de l'annexe montre que chaque variable exogène explique la variable endogène. Cependant les effets de taille varient du faite que les variables digitalisation et support de direction ont un effet important alors que le comportement des demandeurs de crédits et l'intensité de la concurrence ont un effet faible.

La valeur $\mathrm{Q}^{2}$ a été trouvée supérieure à zéro, soit 0,884 pour la digitalisation et 0,937 pour la performance ce qui signifié le modèle est de nature prédictive.

Le calcule l'indice d'ajustement GoF (Goodness-of-fit est basé sur le tableau 3 de l'annexe. La valeur du GoF est égale à 1,3404 ce qui signifié que le modèle est suffisamment large pour la validité globale du modèle PLS (Wetzels et al. (2009)).

\subsection{Analyse du rôle médiateur de la digitalisation}

La situation dans laquelle une variable absorbe dans une certaine mesure l'effet d'une variable exogène (support de la direction) sur une construction endogène (performance de financement) représente la médiation. La variable en question est appelée variable médiatrice. La mesure du rôle médian d'une variable par l'approche PLS est faite par la technique de rééchantillonnage bootstrapping. Pour considérer qu'il y a un effet médian d'une variable Preacher et Hayes (2008), prévoient deux conditions :

La relation entre le support de la direction et la performance de financement doit être significative ( $\mathrm{p}$-value < 0.05) en se basant sur le Bootstrap effet indirect (total effet).

le levier inferieur et le levier supérieur ne doivent pas croiser le 0 en se basant sur le Boostrap Intervalle de confiance.

Pour le Bootstrap effet indirect (total effet), il faut la relation indirect entre support de direction et la performance de financement qui intègre la digitalisation soit significative ce qui représente l'effet indirects spécifiques et que les trois relations entre les variables latentes soient significatives ce qui représente l'effet total. 
Pour l'effet indirects spécifiques, la relation indirecte entre le support de direction et la performance du financement est significative du faite que la $\mathrm{p}$-value tend vers $0(\mathrm{P}=0.000)$ ce qui est équivalent à une statistique $T$ très élévé $(5,905)$. Les résultats sont récapitulés dans le tableau 5 suivant :

Tableau 5 : Effets indirects spécifiques

\begin{tabular}{|r|r|r|r|r|}
\hline & Beta & Écart-type & \multicolumn{1}{c|}{ Valeur T } & \multicolumn{1}{c|}{ valeurs-p } \\
\hline$S D \rightarrow E F \rightarrow P F$ & 0,476 & 0,085 & 5,905 & $\mathbf{0 , 0 0 0}$ \\
\hline
\end{tabular}

Les effets totaux sont significatifs pour les 3 relations avec $\mathrm{p}<0.01$. Les valeurs des betas, des statistiques $\mathrm{T}$ et des $\mathrm{p}$ values sont présentées dans le tableau 4 de l'annexe.

En ce qui concerne le levier inferieur et le levier supérieur, la deuxième condition de Preacher et Hayes (2008) est remplie. En effet, le levier inferieur est égal à 0,344 et le levier supérieur est égal à 0,650 , présenté dans le tableau 5 de l'annexe, ne croisent pas le 0 . En conséquence, la digitalisation joue un rôle médian significatif (Valeur $T=5,905$ ) entre le support de la direction et la performance de financement.

\subsection{Analyse de l'effet modérateur du comportement des demandeurs de crédits à l'égard de la digitalisation.}

Borau et al. (2015) définit le rôle modérateur comme l'existence d'une ou de plusieurs variables qui modulent l'influence d'une variable $\mathrm{X}$ sur une variable $\mathrm{Y}$, en impactant la nature, le sens et/ou la force de cette influence qui varierait selon les valeurs de la variable modératrice.

L'analyse de l'effet modérateur de la variable comportement des demandeurs de crédits à l'égard de la digitalisation $(C D C)$ sur l'influence de la variable digitalisation ( $\mathrm{EF}$ ) sur la variable dépendante performance de financement $(P F)$ à rejeté l'existence d'effet de modération du fait que la p-value égale à 0,228 est supérieure à 0,05 . De fait, l'hypothèse $H_{4}$ est rejetée. Le tableau 6 présente les résultats de l'analyse de l'effet modérateur.

Tableau 6 : Effet modérateur du comportement des demandeurs de crédits

\begin{tabular}{|l|c|r|r|r|}
\hline & Beta & Écart-type & Valeur T & valeurs-p \\
\hline Effet modérateur1 $\rightarrow P F$ & 0,027 & 0,02 & 1,210 & $\mathbf{0 , 2 2 6}$ \\
\hline
\end{tabular}

\subsection{Analyse de l'effet modérateur de l'intensité concurrentielle.}

L'analyse de l'effet modérateur de la variable intensité concurrentielle ( ) sur l'influence de la variable digitalisation ( $\boldsymbol{E} \boldsymbol{F}$ ) sur la variable dépendante performance de financement ( $\boldsymbol{P F}$ ) a rejeté l'existence d'effet de modération du fait que la p-value est supérieur à 0,05 . De fait, l'hypothèse $H_{5}$ est rejetée. Le tableau 7 présente les résultats de l'analyse de l'effet modérateur.

Tableau 7 : Effet modérateur l'intensité concurrentielle.

\begin{tabular}{|l|r|r|r|r|}
\hline & \multicolumn{1}{|l|}{ Beta } & \multicolumn{1}{|l|}{ Écart-type } & Valeur T & valeurs-p \\
\hline Effet modérateur1 $\rightarrow P F$ & 0,040 & 0,023 & 1,740 & $\mathbf{0 , 0 8 1}$ \\
\hline
\end{tabular}

\section{Discussion.}

L'utilisation des modèles d'équations structurelles par l'approche PLS permet de déterminer les relations entre les variables latentes et des modèles de prédictions de l'interaction entre ces variables. En effet, cette démarche est utilisée dans ce papier pour l'apport de la digitalisation dans le processus d'octroi de crédits. 
Les résultats de cette étude montrent que la digitalisation a un impact positif sur la performance de financement. L'analyse effectuée selon la méthode PLS a montré que les coéfficients de régression du modèle structurel sont significatifs du fait qu'ils vérifient les critères de Hair et al. (2014).

Aussi la digitalisation joue un rôle médiateur entre le support de direction et la performance de financement du fait que le modèle vérifié les conditions de Preacher et Hayes (2008).

En ce qui concerne, l'intensité concurrentielle et le comportement des demandeurs de crédits à l'égard de la digitalisation, cette étude a montré que ces deux facteurs n'ont pas d'effet sur la performance de financement et ne modère pas la relation entre le support de direction et la performance de financement.

\section{Conclusion}

Cette étude examine 5 hypothèses de relations positives entre 5 variables latentes à savoir le support de direction, la performance de financement, la digitalisation, l'intensité concurrentielle et le comportement des demandeurs de crédits à l'égard de la digitalisation. Pour vérifier ces hypothèses cette étude utilise les modèles d'équations structurelles avec la méthode PLS.

Les ithems et les variables latentes sont déterminés à partir de la revue de littérature concernant plusieurs domaines de gestion notamment en marketing. Le traitement des données a montré l'existence d'une relation linéaire entre le support de direction, la digitalisation et la performance de financement et rejet l'impact de l'intensité concurrentielle et le comportement des demandeurs de crédits.

Aussi, l'étude a montré que la digitalisation modère la relation entre le support de direction et la performance de financement ce qui signifie l'apport positif de la digitalisation en matière de financement.

Par ailleurs, cette étude utilise un échantillon de faible taille, les résultats constatés notamment pour les deux dernières hypothèses doivent faire l'objet d'une étude future de confirmation moyennant un échantillon de grande taille.

\section{BIBLIOGRAPHIE}

[1] Amin, M. (2016), "Internet banking service quality and its implication on ecustomer satisfaction and ecustomer loyalty", International Journal of Bank Marketing, Vol. 34 No. 3, pp. 280306. https://doi.org/10.1108/IJBM1020140139

[2] Aral,S., Weill,P.(2007). IT Assets, Organizational Capabilities, and Firm Performance: How Resource Allocations and Organizational Differences Explain Performance Variation, Vol. 18, No. 5, pp. 763-780 issn 10477039 | eissn 15265455 |07 |1805 | 0763, doi 10.1287/orsc.1070.0306

[3] Bennaceur, A., Chafik, K. (2019). Les fondements de l'usage des équations structurelles dans les recherches en sciences de gestion: Cas de l'approche PLS. Revue du contrôle, de la comptabilité et de l'audit, 3(2).

[4] Bolos, C., Idemudia, E. C., Mai, P., Rasinghani, M., Smith, S. (2016). Conceptual Models on the Effectiveness of EMarketing Strategies in Engaging Consumers. International Information Management Association

[5] Borau, S., Akremi, A. E., ElgaaiedGambier, L., HamdiKidar, L., Ranchoux, C. (2015). (1. F. Marketing, Éd.) Recherche et Applications en Marketing, 30(04), pp. 95-138.

[6] Brodie, R.J., Winklhofer, H. , Coviello, N.E. and Johnston, W.J. (2007). is emarketing coming of age? an examination of the penetration of emarketing and firm performance. Journal of Interactive Marketing.

[7] Chae, HoChang; Koh, Chang E.; and Prybutok, Victor R..(2014). "Information Technology Capability and Firm Performance: Contradictory Findings and Their Possible Causes," MIS Quarterly, (38: 1) pp.305325. 
[8] Chavey, D. (2010). Applying organisational capabilities models to access the maturity of digital marketing gouvernance. Journal of marketing management, 26(3 4), pp. 187196

[9] Chin W.W. (1998), « The partial least squares approach to structural equation modeling », In G. A. Marcoulides (Ed.), Modern methods for business research, (pp. 295336), Mahwah, NJ: Lawrence Erlbaum.

[10] Chin, W. W., Newsted, P. R. (1999). Structural equation modeling analysis with small samples using partial least squares. In R. H. Hoyle (Ed.), Statistical strategies for small sample research (pp. 307341). Thousand Oaks: CA: Sage Publications.

[11] Shinta Winasis, Uli Wildan, Achmad H. Sutawidjaya (2020), Impact of Digital Transformation onEmployee Engagement Influenced by Work Stress on Indonesian Private Banking Sector, Proceedings of the 5th NA International Conference on Industrial Engineering and Operations Management Detroit, Michigan, USA, August 10 14, 2020

[12] Deltour, F., Lethiais, V. (2014). L'innovation en PME et son accompagnement par les TIC : quels effets sur la performance ? Systèmes d'information management, 19, pp. p. 4573

[13] Davcik, N. S. (2014). The use and misuse of structural equation modeling in management research: A review and critique, Journal of Advances in Management Research, Vol. 11 No. 1 , pp. 4781. https://doi.org/10.1108/JAMR0720130043

[14] Eyup Kahveci and Bert Wolfs (2018). Digital banking impact on Turkish deposit banks performance. Banks and Bank Systems, 13(3), 4857. doi:10.21511/bbs.13(3).2018.05

[15] Faliagka, E.; Ramantas, K.; Tsakalidis, A.; Viennas, M.; Kafeza, E. and Tzimas, G. (2011). AN INTEGRATED ERECRUITMENT SYSTEM FOR CV RANKING BASED ON AHP. In Proceedings of the 7th International Conference on Web Information Systems and Technologies WEBIST, ISBN 9789898425515; ISSN 21843252, pages 147150. DOI: $10.5220 / 0003337901470150$

[16] Fernandes V. (2012). En quoi l'approche PLS estelle une méthode à (re)découvrir pour les chercheurs en management? M@n@gement, 15,1, 101123.

[17] Fornell, C. and Larcker, D.F. (1981). Evaluating Structural Equation Models with Unobservable Variables and Measurement Error, Journal of Marketing Research, Vol. 18, No. 1 (Feb., 1981), pp. 3950 (12 pages), https://doi.org/10.2307/3151312

[18] Germann, F.,1 , Lilien, G.L., Rangaswamy A. (2013). Performance implications of deploying marketing analytics, International Journal of Research in Marketing, Volume 30, Issue 2, June 2013, Pages 114128.

[19] Hair, J. F., Sarstedt, M., Hopkins, L., Kuppelwieser, V. G. (2014). Partial least squares structural equation modeling (PLSSEM) An emerging tool in business research. European Business Review, 26(2), pp. 106121.

[20] Höck, M. and Ringle, C.M. (2006). Local strategic networks in the software industry: An empirical analysis of the value continuum, International Journal of Knowledge Management Studies 4(2). DOI:10.1504/IJKMS.2010.030789

[21] Jaworski, B. J., Kohli, A. L. (1993). Market Orientation: Antecedents and Consequences. Journal of Marketing(57), pp. 5371

[22] Jörg, H., Marko, S. (2013). Goodnessoffit indices for partial least squares path modeling. Comput Stat, 28, pp. 565-580.

[23] Kannan, P., Hongshuang, L. A. (2017). Digital marketing: A framework, review and research agenda. International Journal of Research in Marketing, 34, pp. 2245

[24] Kitsios, F.; Giatsidis, I.; Kamariotou, M. Digital Transformation and Strategy in the Banking Sector: Evaluating the Acceptance Rate of EServices. J. Open Innov. Technol. Mark. Complex. 2021, 7, 204. https://doi.org/10.3390/ joitmc7030204.

[25] MBAMA, Cajetan (2018). Digital banking, customer experience and bank financial performance : UK customers' perceptions. International Journal of Bank Marketing, 36 (2), 230255.

[26] Morgan, N. A. (2012). Marketing and business performance. Journal of the Academic Marketing Science, pp. 102119.

[27] Ismail Musabegović, Mustafa Ozer and Stefan Jovanović (2021), INTERPLAY BETWEEN DIGITAL TECHNOLOGIES AND BUSINESS PERFORMANCE IN BANKING INDUSTRY: GLOBAL AND REGIONAL PERSPECTIVES, Ekonomika . JulSep2021, Vol. 67 Issue 3, p7592. 18p.

[28] Meher, B. K., Hawaldar, I. T., Mohapatra, L., Spulbar, C., Birau, R., \& Rebegea, C. (2021). The impact of digital banking on the growth of Micro, Small and Medium Enterprises (MSMEs) in India: a case study. Business: Theory and Practice, 22(1), 1828. https://doi.org/10.3846/btp.2021.12856 Olomu, M. O., Irefin, I. A. (2016). Emarketing adoption and performance in the nigerian paints industry. International Journal of Economics, Commerce and Management.

[29] Piening, E. P., Baluch, A. M., Salge, T. O. (2013). The relationship between employees' perceptions of human resource systems and organizational performance: Examining mediating mechanisms and temporal dynamics. Journal of Applied Psychology, 98(6), 926-947. https://doi.org/10.1037/a0033925 
[30] Porter, M. E., Millar, V. A. (1985). How Information Gives You Competitive Advantage. Harvard Business Review, 63(4), pp. 149160.

[31] Preacher, K. J., Hayes, A. F. (2008). Contemporary approaches to assessing mediation in communication research. In A. F. Hayes, M. D. Slater, L. B. Snyder (Eds.), The Sage sourcebook of advanced data analysis methods for communication research (pp. 13-54). Sage Publications, Inc. https://doi.org/10.4135/9781452272054.n2

[32] Rafiq Q.A., Brosnan, K.M., Coopman, K., Nienow, A.W., Hewitt, C.J. ,(2013). Culture of human mesenchymal stem cells on microcarriers in a 51 stirredtank bioreactor", Biotechnology letters, 35, 12331245.

[33] Rila Anggraeni, Raditha Hapsarib Noor Awanis Muslim (2021), Examining Factors Influencing Consumers Intention and Usage of Digital Banking: Evidence from Indonesian Digital Banking Customers, AsiaPacific Management and Business Application, 9, 3 (2021): 193210, DOI: 10.21776/ub.apmba.2021.009.03.1.

[34] Roussel P. (2005). Méthodes de développement d'échelles pour questionnaires d'enquête - in « Management des ressources humaines, méthodes de recherches en sciences humaines et sociales »; sous la direction de Roussel P. Wacheux F. - Bruxelles, De Boeck

[35] Sheikh, A.A., Rana,N.A. Aneeq I., Arfan S. Hayat Muhammad Awan, H.M. (2018). Is emarketing a source of sustainable business performance? Predicting the role of top management support with various interaction factors, Cogent Business Management, 5:1, DOI: 10.1080/23311975.2018.1516487

[36] Trainor, K., Rapp , A., Skinner Beitelspacher, L., Schillewaert, N. (2011). Integrating information technology and marketing: An examination of the drivers and outcomes of eMarketing capability. Industrial Marketing Management

[37] Wetzels, M., OdekerkenSchröder, G., Van Oppen, C. (2009). Using PLS path modeling for assessing hierarchical construct models: guidelines and empirical illustration. MIS Quarterly, 33(1), 177-195. https://doi. org/10.2307/20650284

[38] Wold, H. (1975). Soft modeling by latent variables: The Non - Linear Iterative Partial Least Squares (NIPALS) approach, in Gani, J. (Editor), Perspectives in probability and statistics, pages 117 - 142, Londres

[39] Wold, H. (1985). Partial Least Squares, Kotz, S. et Johnson, N. L. (Editors), Encyclopedia of Statistical Sciences, Vol. 6, pages 581591, John Wiley Sons, New York

[40] Wold, H., (1973). Nonlinear iterative partial least squares (NIPALS) modelling. Some current developements. In : Krishnaiah, P.R. (Ed.), Multivariate Analysis, Vol. III. Academic Press, New York, pp. 383-407. 17, 20, 117

[41] Wold, H., (1980a). The fixpoint approach to interdependent systems. North Holland, Amsterdam. 20, 49

[42] Wold, H., (1980b). Model construction and evaluation when theoretical knowledge is scarce. In : Kmenta, J., Ramsey, J.B. (Eds.), Evaluation of econometric models. Academic Press, pp. 47-74. 20, 57

[43] Zhu, K., Kraemer, K. L., Xu, S. (2006). The Process of Innovation Assimilation by Firms in Different Countries: A Technology Diffusion Perspective on EBusines. Management sciences, 52(10), pp. 15571576.

[44] Zuo, L.; Strauss, J.; Zuo, L. The Digitalization Transformation of Commercial Banks and Its Impact on Sustainable Efficiency Improvements through Investment in Science and Technology. Sustainability 2021, 13, 11028. https://doi.org/10.3390/ su131911028;

ANNEXE 1 :

Table 1 : Validité discriminante- chargement croisé (cross loading)

\begin{tabular}{|c|c|c|c|c|c|}
\hline & CDC & EF & IC & PERFO & SUPDIR \\
\hline CD1 & $\mathbf{0 , 9 0 2}$ & 0,585 & 0,812 & 0,537 & 0,557 \\
\hline CD2 & $\mathbf{0 , 9 1 5}$ & 0,541 & 0,725 & 0,506 & 0,529 \\
\hline CD3 & $\mathbf{0 , 8 5 0}$ & 0,725 & 0,845 & 0,646 & 0,689 \\
\hline EF1 & 0,693 & $\mathbf{0 , 9 0 1}$ & 0,819 & 0,870 & 0,864 \\
\hline EF2 & 0,604 & $\mathbf{0 , 8 2 5}$ & 0,740 & 0,800 & 0,780 \\
\hline EF3 & 0,654 & $\mathbf{0 , 7 9 9}$ & 0,750 & 0,767 & 0,744 \\
\hline EF4 & 0,647 & $\mathbf{0 , 8 3 0}$ & 0,748 & 0,804 & 0,789 \\
\hline EF5 & 0,613 & $\mathbf{0 , 9 6 3}$ & 0,874 & 0,930 & 0,809 \\
\hline EF6 & 0,520 & $\mathbf{0 , 8 4 0}$ & 0,608 & 0,814 & 0,767 \\
\hline IC1 & 0,749 & 0,709 & $\mathbf{0 , 9 1 8}$ & 0,677 & 0,707 \\
\hline IC2 & 0,833 & 0,740 & $\mathbf{0 , 8 8 8}$ & 0,679 & 0,707 \\
\hline IC3 & 0,826 & 0,746 & $\mathbf{0 , 9 1 6}$ & 0,743 & 0,778 \\
\hline PR1 & 0,595 & 0,883 & 0,736 & $\mathbf{0 , 9 0 1}$ & 0,893 \\
\hline
\end{tabular}


Int. J. Econ. Stud. Manag. 1, No.3, 338-353 (December-2021)

\begin{tabular}{|c|l|l|l|l|l|}
\hline PR2 & 0,661 & 0,908 & 0,760 & $\mathbf{0 , 9 3 2}$ & 0,906 \\
\hline PR3 & 0,500 & 0,853 & 0,656 & $\mathbf{0 , 9 1 7}$ & 0,868 \\
\hline S1 & 0,606 & 0,902 & 0,773 & 0,904 & $\mathbf{0 , 9 1 8}$ \\
\hline S2 & 0,671 & 0,877 & 0,777 & 0,884 & $\mathbf{0 , 9 0 3}$ \\
\hline S3 & 0,693 & 0,876 & 0,802 & 0,900 & $\mathbf{0 , 9 2 6}$ \\
\hline S4 & 0,523 & 0,820 & 0,678 & 0,840 & $\mathbf{0 , 8 6 1}$ \\
\hline
\end{tabular}

Tableau 2 : Les valeurs de $F_{k}^{2}$

\begin{tabular}{|c|c|c|c|}
\hline & EF & PF & Résultat \\
\hline EF & & 0,916 & Effet important \\
\hline SD & 8,722 & 1,032 & Effet important \\
\hline CDC & & 0,041 & Effet faible \\
\hline IC & & 0,006 & Effet faible \\
\hline
\end{tabular}

Tableau 3 : élément de calcul du GOF

\begin{tabular}{|c|c|c|}
\hline Variable latente & $A V E=H^{2}$ & $\mathrm{R}^{2}$ \\
\hline SD & 0,812 & 0,877 \\
\hline EF & 0,949 & 0,988 \\
\hline PF & 0,868 & \\
\hline CDC & 0,865 & \\
\hline IC & 0,828 & 0,9325 \\
\hline Moyenne & 0,8644 & \\
\hline
\end{tabular}

GoF $=\sqrt{\overline{H^{2}} \times \overline{R^{2}}}=1,3404$

Tableau 4 : Effets totaux

\begin{tabular}{|l|r|r|r|r|r|}
\hline & Beta & Écart-type & Valeur $T$ & valeurs-p & Décision \\
\hline $\mathrm{EF} \rightarrow \mathrm{PF}$ & 0,504 & 0,086 & 5,990 & $\mathbf{0 , 0 0 0}$ & Supported* \\
\hline $\mathrm{SD} \rightarrow \mathrm{EF}$ & 0,924 & 0,014 & 67,925 & $\mathbf{0 , 0 0 0}$ & Supported* $^{*}$ \\
\hline $\mathrm{SD} \rightarrow \mathrm{PF}$ & 1,032 & 0,033 & 31,751 & $\mathbf{0 , 0 0 0}$ & Supported* $^{*}$ \\
\hline
\end{tabular}

$* \mathrm{p}<0.01$

Tableau 5 : levier inferieur et le levier supérieur

\begin{tabular}{|l|l|l|l|c|l|l|}
\hline $\mathrm{SD} \rightarrow \mathrm{EF}$ & $\mathrm{EF} \rightarrow \mathrm{PF}$ & Effet indirect & Ecart type & $T$ & LL & UL \\
\hline 0,924 & 1,032 & 0,476 & 0,086 & 5,905 & 0,344 & 0,650 \\
\hline
\end{tabular}

